\title{
A Soundness Test for Multi Criteria Decision Models with Imprecise Data
}

\author{
${ }^{*}$ Camilo Franco $^{a}$ and Jens Leth Hougaard ${ }^{b}$ \\ ${ }^{a}$ Department of Industrial Engineering, Faculty of Engineering, University of the Andes, 111711 Bogota, \\ c.franco31@uniandes. edu.co \\ ${ }^{b}$ Department of Food and Resource Economics, Faculty of Economics, University of Copenhagen, 1870 Frederiksberg, \\ jlh@ifro.ku.dk
}

\begin{abstract}
This paper proposes a set of minimal conditions which should be fulfilled by any multi-criteria additive procedure set out to work under imprecision. The proposed conditions are defined in terms of binary preference relations, which are inferred from interval-valued alternatives, and are shown to be useful for evaluating the intervalbased reasoning procedures of imprecise multi-criteria methods. In particular, wellknown distance-based methods like TOPSIS and VIKOR are examined in their interval extensions, together with the interval-valued method of WOD, finding that only VIKOR fails to pass the complete test.
\end{abstract}

Keywords: Imprecision, Interval-valued data, Preference estimation

\section{Introduction}

Decision modeling is a fundamental field for explaining and solving real world problems, where uncertainty and imprecision emerge as key aspects for properly representing the knowledge contained in common observations and measurements. Addressing imprecision, a set of values, equipped with a lower and an upper bound, is considered to represent the suitability of alternatives according to a given set of criteria, instead of an exact or unique value (see e.g. $[5,6,9])$. Accordingly, the objective here is to reflect on how preferences are actually being inferred by multi-criteria procedures under imprecision.

Examining preference estimation in multi-criteria decision modeling (MCDM) [2], its various methods aim at identifying a pre-order (if possible) over the available alternatives. For doing so, preferences have to be firstly estimated, comparing in a pairwise manner the alternatives, which are valued by interval scores. The different interval-valued multi criteria methods examined in this study consist in the interval extension of distance-based methods Technique for Order of Preference by Similarity to Ideal Solution (TOPSIS) [4, 8] and Multicriteria Optimization and Compromise Solution (VIKOR, proposed in [10, 11], and extended for interval data in $[6,15])$, and the Weighted Overlap Dominance method (WOD, as presented in [7]).

We show that extended versions of TOPSIS as well as the WOD method satisfy the complete relational test, while VIKOR fails to do so unless an adequate tuning of its free parameters. Using these latter methods, the analyst should therefore be careful in the specific procedure for comparing interval numbers with the VIKOR method. Otherwise the consistency of the results will be at stake.

In order to bring forward our reflection on imprecise MCDM and the proposed tests for imprecise preference estimation, the first section presents some preliminaries and definitions, introducing the multi-criteria decision problem setting. In Section 3 , the multi criteria methods are reviewed, namely TOPSIS, VIKOR, and the WOD method. In Section 4 the reasoning framework is presented for a formal definition of three dominance tests and in Section 5 we assess the different methods under the light of the proposed tests. Lastly, some conclusions are presented together with final comments for future research.

\section{Preliminaries}

In this section we present the setting for examining imprecise multi-criteria decision problems and some definitions that are going to be used in the paper. 


\subsection{Imprecise-MCDM setting}

The preference estimation stage for imprecise-MCDM focuses on estimating pairwise preference relations from interval data. For this purpose, consider the general class of multiple criteria decision problems, involving a (non-empty) finite set of alternatives $N$, such that $|N|=n$, and a finite set of criteria $M$, such that $|M|=m$. Alternatives are evaluated considering each criterion, and the result of such an evaluation is aggregated among all (weighted) criteria, usually as a weighted average with respect to a given vector of weights $w \in[0,1]^{m}$. These weights express the relative importance of criteria, and are used for the estimation of a global preference order.

Under a precise setting, each alternative $i \in N$ can be valued according to a given criterion $j \in M$, by some degree $\mu_{i j} \in \mathscr{L}$, e.g. $\mathscr{L}=[0,1]$, which can be understood according to a fuzzy set and its membership function (see [14]), expressing the extent up to which alternative $i$ fulfills criterion $j$ (see e.g. [2]). Now, taking into account some natural imprecision on the exact value of such a degree, an interval scale

$$
\mathscr{L}=\{[x, y] \subseteq[0,1] \mid x \leq y\}
$$

can be used to assign an approximate set of values which contain the true value, expressed by a pair of exact lower and upper bounds. Thus, the suitability of alternatives with respect to the available criteria is expressed by an interval-valued degree.

In consequence, an interval-valued degree is taken here to represent $(\forall i \in N, \forall j \in M)$ the suitability of alternatives, given by

$$
\mu_{i j}=\left[\mu_{i j}^{L}, \mu_{i j}^{U}\right],
$$

where $\mu_{i j}^{L}$ and $\mu_{i j}^{U}$ are the lower and upper bounds of $\mu_{i j}$, respectively.

In this way, the complete $(n \times m)$ data matrix for this general class of multi-criteria decision problems is given by

$$
\boldsymbol{A}=\left(\begin{array}{ccc}
{\left[\mu_{11}^{L}, \mu_{11}^{U}\right]} & \ldots & {\left[\mu_{1 m}^{L}, \mu_{1 m}^{U}\right]} \\
\vdots & \ddots & \vdots \\
{\left[\mu_{n 1}^{L}, \mu_{n 1}^{U}\right]} & \ldots & {\left[\mu_{n m}^{L}, \mu_{n m}^{U}\right]}
\end{array}\right) .
$$

The formulation of the problem according to (1) focuses on methods which receive as input the imprecise valuations for each alternative regarding each criterion, estimate binary preference relations, and return as output a preference order for the alternatives.
Before reviewing the multi-criteria methods that are going to be included in this study, a couple of key relational situations among alternatives $i, k \in N$ have to be pointed out. On the one hand, alternatives $i$ and $k$ are said to be strictly equivalent according to the following definition.

Definition 1. [5] For all $(i, k) \in N \times N$, given a vector of weights $w \in[0,1]^{m}$ and decision matrix $(1),(i, k)$ is a strictly equivalent pair if, and only if,

$$
w \cdot \mu_{i}^{U}=w \cdot \mu_{k}^{U}
$$

and

$$
w \cdot \mu_{i}^{L}=w \cdot \mu_{k}^{L} .
$$

In consequence, any pair of equivalent alternatives $(i, k) \in N \times N$ verifies an indifferent relation $(\sim)$, being defined as the absence of strict preference $(\succ)$, such that $i \sim k$.

On the other hand, alternatives $i$ and $k$ are said to be partially equivalent in the following sense.

Definition 2. For all $(i, k) \in N \times N$, given a vector of weights $w \in[0,1]^{m}$ and decision matrix $(1),(i, k)$ is a partially equivalent pair if, and only if,

$$
w \cdot \mu_{i}^{U}=w \cdot \mu_{k}^{U} \text { and } w \cdot \mu_{i}^{L} \neq w \cdot \mu_{k}^{L}
$$

or

$$
w \cdot \mu_{i}^{L}=w \cdot \mu_{k}^{L} \text { and } w \cdot \mu_{i}^{U} \neq w \cdot \mu_{k}^{U}
$$

Definitions 2-2 illustrate simple cases that can be directly identified, upon which we will develop our analysis motivating the set of relational specific situations that are going to be used for assessing preference estimation under imprecision. For simplicity, throughout the paper we will take the condition of partial equivalence according to Eq. (4).

\section{Imprecise-MCDM}

In this section the MCDM methods that are going to be used on this study are reviewed.

\subsection{Distance-based methods}

Different multi-criteria methods, such as TOPSIS [8] or VIKOR $[13,10]$, depend on the computation of the distance of alternatives to some reference points, or ideally best and worst solutions. These reference points are taken in the TOPSIS $[1,3]$ as the maximum and minimum scores for each criterion, known as the Positive Ideal Solution (PIS), and the Negative Ideal Solution (NIS), respectively. Now, following the interval fuzzy TOPSIS extension(s) (see e.g. [1, $3]$ ), distances have to be defined for interval fuzzy 
values (in fact, TOPSIS was initially extended to interval values in [8]), with the purpose of defining relative closeness indexes to the positive and negative references.

For this purpose, take any distance function, like e.g. the weighted L-1 measure defined for any pair of interval-valued multi-criteria scores $\mu_{i}$ and $\mu_{k}$, and weights $w \in \mathbb{R}^{m}$, by

$$
w \cdot d\left(\mu_{i}, \mu_{k}\right)=\sum_{j=1}^{m} w_{j}\left(\left|\mu_{i j}^{U}-\mu_{k j}^{U}\right|+\left|\mu_{i j}^{L}-\mu_{k j}^{L}\right|\right) .
$$

Then, the relative closeness indexes of the TOPSIS can be computed according to $D_{i}^{+}$and $D_{i}^{-}$, respectively denoting the distance of any alternative $i \in N$ to the corresponding positive and negative references. Such references are here fixed for given positive and negative prototypes, respectively given by $\mu^{+}=$ $\left(\mu_{+}^{L}, \mu_{+}^{U}\right)=\left(\left\{\mu_{+1}^{L}, \ldots, \mu_{+m}^{L}\right\},\left\{\mu_{+1}^{U}, \ldots, \mu_{+m}^{U}\right\}\right) \quad$ and $\mu^{-}=\left(\mu_{-}^{L}, \mu_{-}^{U}\right)=\left(\left\{\mu_{-1}^{L}, \ldots, \mu_{-m}^{L}\right\},\left\{\mu_{-1}^{U}, \ldots, \mu_{-m}^{U}\right\}\right)$. For simplicity, take e.g. $\mu^{+}=\mathbf{1}$ and $\mu^{-}=\mathbf{0}$.

Therefore, alternatives are ordered according to their relative closeness indexes $R C_{i}$, such that [1]

$$
R C_{i}=\frac{D_{i}^{-}}{D_{i}^{+}+D_{i}^{-}}
$$

where the higher the index, the better the alternative, as it expresses how far away it is from the worst possible solution. Then, understanding this closeness index as the intensity of (a distance-based) preference, a preference relation can be defined in a pairwise manner $(\forall i, k \in N)$, such that, if $R C_{i}>R C_{k}$, then $i \succ k$ holds, and in case $R C_{i}=R C_{k}$, then $i \sim k$ holds true.

Notice that by using the distance measure (6), the resulting preference relation is uniquely valued, as the relative closeness indexes obtain a unique value for the ratio (7). On the other hand, if we define a distance measure for interval values that computes separately upper bounds from lower bounds, then the result would be an interval valued preference.

Such an approach can be examined under the VIKOR method (introduced in 1998, as pointed out in [10]), which also computes distances for each $i \in N$ to the PIS and NIS, but distinguishing upper from lower bounds. In this way, the VIKOR method computes an average and a maximum closeness to the ideal points, respectively given by $A_{i}$ and $M_{i}$. Then, an overall (compromise solution [13]) index $Q_{i}$ is obtained for ordering the alternatives, which for the interval extensions $[15,6,12]$, is in fact an interval-valued index $Q_{i}=\left[Q_{i}^{L}, Q_{i}^{U}\right]$. In this sense, the alternatives' preference is understood according to their relative closeness to the PIS and the NIS, defined here, for $i=1, \ldots, n$, as

$$
\begin{aligned}
& A_{i}^{L}=\sum_{j \in I} w_{j}\left(\frac{\mu_{j}^{P}-\mu_{i j}^{U}}{\mu_{j}^{P}-\mu_{j}^{N}}\right)+\sum_{j \in J} w_{j}\left(\frac{\mu_{i j}^{L}-\mu_{j}^{P}}{\mu_{j}^{N}-\mu_{j}^{P}}\right) \\
& A_{i}^{U}=\sum_{j \in I} w_{j}\left(\frac{\mu_{j}^{P}-\mu_{i j}^{L}}{\mu_{j}^{P}-\mu_{j}^{N}}\right)+\sum_{j \in J} w_{j}\left(\frac{\mu_{i j}^{U}-\mu_{j}^{P}}{\mu_{j}^{N}-\mu_{j}^{P}}\right)
\end{aligned}
$$

and

$$
\begin{aligned}
M_{i}^{L}= & \max \left\{w_{j}\left(\frac{\mu_{j}^{P}-\mu_{i j}^{U}}{\mu_{j}^{P}-\mu_{j}^{N}}\right) \mid j \in I,\right. \\
& \left.w_{j}\left(\frac{\mu_{i j}^{L}-\mu_{j}^{P}}{\mu_{j}^{N}-\mu_{j}^{P}}\right) \mid j \in J\right\} \\
M_{i}^{U}= & \max \left\{w_{j}\left(\frac{\mu_{j}^{P}-\mu_{i j}^{L}}{\mu_{j}^{P}-\mu_{j}^{N}}\right) \mid j \in I,\right. \\
& \left.w_{j}\left(\frac{\mu_{i j}^{U}-\mu_{j}^{P}}{\mu_{j}^{N}-\mu_{j}^{P}}\right) \mid j \in J\right\}
\end{aligned}
$$

where $\mu_{j}^{P}$ and $\mu_{j}^{N}$ respectively represent the PIS and the NIS, $I$ is the set of beneficial criteria and $J$ is the set of costly criteria.

Then, alternatives are ranked according to the average interval-valued index $Q_{i}=\left[Q_{i}^{L}, Q_{i}^{U}\right]$, where the lower the index, the better the alternative. Thus, the best alternative is the one that obtains the minimum value for $Q_{i}$, given $(\forall i \in N)$ by

$$
Q_{i}^{L}=v \frac{A_{i}^{L}-A^{*}}{A^{-}-A^{*}}+(1-v) \frac{M_{i}^{L}-M^{*}}{M^{-}-M^{*}}
$$

and

$$
Q_{i}^{U}=v \frac{A_{i}^{U}-A^{*}}{A^{-}-A^{*}}+(1-v) \frac{M_{i}^{U}-M^{*}}{M^{-}-M^{*}},
$$

where $A^{*}=\min _{i} A_{i}^{L}, M^{*}=\min _{i} M_{i}^{L}, A^{-}=\max _{i} A_{i}^{U}$ and $M^{-}=\max _{i} M_{i}^{U}$, and $v$ is the coefficient of a linear (additive) combination between the average and the maximum (aggregation strategies). Here, pairwise preference relations can be estimated $(\forall i, k \in N)$, according to a specific procedure that allows handling interval scores.

Therefore, following its interval extension [12], we have to consider different relative situations between any pair $\left(Q_{i}, Q_{k}\right)$. Firstly consider the case where the interval scores have no intersection. Then the minimum score is the one that has lower values, such that $Q_{i}^{U}<Q_{k}^{L}$ imply that $Q_{i}<Q_{k}$, and hence it holds that $i \succ k$. Another trivial case is the one where two interval scores are the same, such that $Q_{i}^{U}=Q_{k}^{U}$ and $Q_{i}^{L}=Q_{k}^{L}$. Then it is said that $i \sim k$. Finally there are two more cases regarding the interval nature of the 
comparison between $Q_{i}$ and $Q_{k}$, for some $0<\alpha \leq 1$. On the one hand, in the case that $Q_{i}^{L} \leq Q_{k}^{L}<Q_{k}^{U} \leq Q_{i}^{U}$, then $Q_{i}<Q_{k}$ holds true if $\alpha\left(Q_{k}^{L}-Q_{i}^{L}\right) \geq(1-\alpha)\left(Q_{i}^{U}-\right.$ $\left.Q_{k}^{U}\right)$, otherwise $Q_{i}>Q_{k}$. And on the other hand, in the case that $Q_{i}^{L}<Q_{k}^{L}<Q_{i}^{U}<Q_{k}^{U}$, then $Q_{i}<Q_{k}$ holds true if $\alpha\left(Q_{k}^{L}-Q_{i}^{L} \geq(1-\alpha)\left(Q_{k}^{U}-Q_{i}^{U}\right)\right.$, otherwise $Q_{i}>Q_{k}$.

Regarding the above mentioned distance-based methods, it should be pointed out that the relational situation that should follow from such a set of rules is not as trivial as it could seem. Let's examine the following example to illustrate issues that deserve close attention, as they can be problematic for proper interval-valued multi-criteria preference modeling. The main issue is that assuming an equivalent framework for treating precisely and imprecisely valued data leads to unexpected results regarding some specific situations.

Example 1. Consider a relational situation among alternatives $i, k, l \in N$, where $i$ and $k$ are partially equivalent, and $l$ contains higher imprecision than $k$. Also let $k$ to have more imprecision than $i$ on one criterion $j$, and $l$ to have more imprecision than $k$ on that same criterion $j$. The interval scores for the three alternatives are given in Table 1.

Table 1: Interval values for partially equivalent alternatives $i$ and $k$ and a third alternative $l$.

\begin{tabular}{lll}
\hline & $j$ & $g$ \\
\hline$i$ & {$[0.6,0.8]$} & {$[0.6,0.8]$} \\
$k$ & {$[0.4,0.8]$} & {$[0.6,0.8]$} \\
$l$ & {$[0.1,1.0]$} & {$[0.6,0.8]$} \\
\hline
\end{tabular}

For our purposes, the computation of the $Q_{i}$ scores from Eqs. (12) can be developed by taking the $v$ coefficient as $v=1$, and $\alpha=0.5$. As a result, we obtain that $Q_{i}=[0.18,0.54], Q_{k}=[0.18,0.72]$ and $Q_{l}=[0,1]$, and therefore, due to the fact that $Q_{i}<Q_{k}, Q_{k}<Q_{l}$ and $Q_{l}<Q_{i}$, it holds that $i \succ k, k \succ l$ and $l \succ i$. But this is an cyclical/intransitive result, following the chain of strict preference relations where l should not be expected to be strictly preferred to $i$.

In consequence, the specific pairwise preference outcome of these interval methods depends entirely on the specific procedure that is used for ranking interval numbers (although the TOPSIS method overcomes this aspect by means of a uniquely valued distance measure for interval scores). Hence, the analyst or decision maker has to determine how to obtain coherent results, by defining a different set of rules or parameters (under the given rules) for comparing interval numbers with the VIKOR method.

One last method that is particularly conceived for interval data is examined next, initially presented in [7] and further developed in [5], inferring preference relations by pairwise Weighted Overlap Dominance (WOD).

\subsection{Weighted Overlap Dominance (WOD)}

Given the input data matrix (1) and criteria weights $w \in[0,1]^{m}$, the WOD method develops a pairwise comparison process, inferring for every $(i, k \in N)$, the estimated value of preference $R(i, k) \in[0,1]$, regarding the type of overlapping situation that exists among interval-valued alternatives.

In this way, for every pair of interval degrees $\mu_{i}, \mu_{k} \in$ $\mathscr{L}$, there are three types of overlapping situations that can hold, namely no overlap, partial overlap, or complete overlap. For a non-overlapping situation, it holds that

$$
w \cdot \mu_{i}^{L}>w \cdot \mu_{k}^{U},
$$

being there total confidence (with respect to the averaging/compensating principle of the weighted sum) in stating that $a \succ b$, thus being true that $R(i, k)=$ 1.

For a partial overlapping situation, it holds that

$$
w \cdot \mu_{i}^{U} \geq w \cdot \mu_{k}^{U} \geq w \cdot \mu_{i}^{L} \geq w \cdot \mu_{k}^{L}
$$

where the estimation of a preference relation in fact depends on the likelihood that one alternative is preferred to the other one. Here, the likelihood estimate is based on a volume operator $V$, such that $V(\emptyset)=0$, and $\forall i \in N$,

$$
V(i)=\prod_{j=1}^{m} w_{j}\left(\mu_{i j}^{U}-\mu_{i j}^{L}\right),
$$

and is computed, assuming a uniform distribution of possible outcomes over the intervals, as in $(\forall i, k \in N)$

$$
R(i, k)=\frac{V\left(Z_{i k}^{U}\right)}{V(i)}+\frac{V\left(Z_{i k}^{O}\right)}{V(i)} \frac{V\left(Z_{k i}^{L}\right)}{V(k)},
$$

where

$$
\begin{gathered}
\left.Z_{i k}^{U}=\left\{\mu_{i}^{L} \leq x \leq \mu_{i}^{U} \mid w \cdot x>w \cdot \mu_{k}^{U}\right)\right\}, \\
\left.Z_{i k}^{O}=\left\{\mu_{k}^{L} \leq x \leq \mu_{k}^{U} \mid w \cdot x>w \cdot \mu_{i}^{L}\right)\right\}, \\
\left.Z_{i k}^{L}=\left\{\mu_{i}^{L} \leq x \leq \mu_{i}^{U} \mid w \cdot x<w \cdot \mu_{k}^{L}\right)\right\} .
\end{gathered}
$$

Notice that in order to have a proper estimation for the likelihood of preference, the subsets $\left\{Z^{U}, Z^{O}, Z^{L}\right\}$ have to be obtained, which is here done after finding the solution for the convex hull in the hyperplane, as the smallest convex set containing the limit points for each case in (18)-(20). 
In this way, computing the volume of the multidimensional interval-valued degree for each alternative, and estimating the pairwise preferences according to (17), $R(i, k)$ expresses the intensity in which strict preference of $i$ over $k$ can be affirmed.

Regarding the last type of overlapping, which is referred to as complete overlapping, it is defined such that

$$
w \cdot \mu_{i}^{L}<w \cdot \mu_{k}^{L} \leq w \cdot \mu_{k}^{U} \leq w \cdot \mu_{i}^{U} .
$$

In this case, the computation of the likelihood of obtaining an outcome from $i$ which surely dominates $k$, is given $(\forall i, k \in N)$ by the likelihood ratio between $i$ dominating $k, \frac{V\left(Z_{i k}^{U}\right)}{V(i)}$, and $k$ dominating $i, \frac{V\left(Z_{i k}^{L}\right)}{V(i)}$, such that

$$
R(i, k)=\frac{V\left(Z_{i k}^{U}\right)}{V\left(Z_{i k}^{L}\right)} .
$$

Therefore, the WOD procedure examines the three above mentioned overlapping scenarios. If no overlap can be verified, a strict preference situation is assigned to $i$ over $k$. Under partial overlapping, strict preference of $i$ over $k$ holds if $R(i, k)$ is greater than some threshold $\beta \in[0,1]$, otherwise alternatives are said to be indifferent. And for complete overlap, $i$ is preferred to $k$ if $R(i, k)$ is greater than some threshold $\gamma \in \mathbb{R}^{+}, k$ is strictly preferred to $i$ if $R(i, k)<\gamma$, and in case that $R(i, k)=\gamma$, both alternatives are said to be indifferent.

Next, we introduce the conditions for checking the soundness of the imprecise reasoning procedures of MCDM methods.

\section{Soundness test for imprecise MCDM}

In this section we suggest three conditions which should be satisfied by any additive procedure set out to estimate pairwise preferences from interval data. As such, these conditions enable a direct evaluation of multi-criteria methods with respect to their reasoning processes under imprecision.

Consider a first case given by a pair of equivalent alternatives, where both alternatives have the same amount of imprecision (formally defined in [5], as the $\varepsilon$-bounded length of the intervals). Now, taking a pair of criteria $j, g \in M$, if both alternatives are equivalent under the specified weights $w^{\prime}=\left\{w_{j}, w_{g}\right\}$, then they can be considered to be indifferent $(\sim)$, being in the relative situation that is depicted in Fig. 1a.

Now, considering a third alternative $l \in N$ with the same amount of imprecision as $i$ and $k$, it should be expected that, $i$ and $k$ being equivalent, strict preference $(\succ)$ holds for $i$ over $l$ if, and only if, $k$ is also

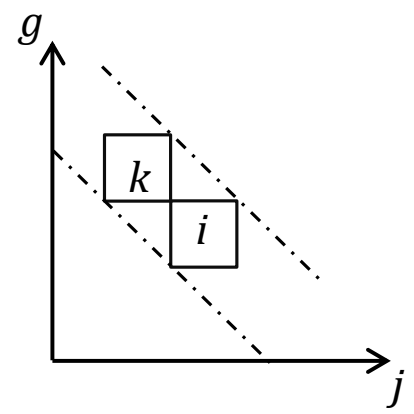

(a)

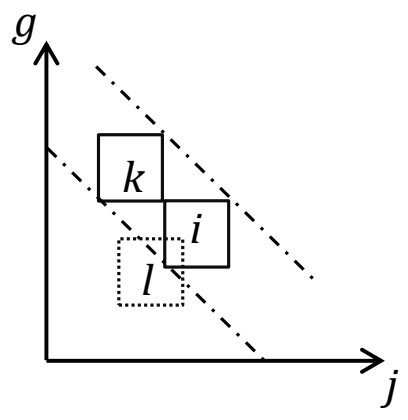

(b)

Figure 1: Relative situation for (a) a pair of equivalent alternatives and (b) a third non-preferred alternative.

strictly preferred to $l$. This is the first condition that should be satisfied by any process aiming at building pairwise preference relations, illustrated in Fig. $1 b$.

Condition 1. Given a pair of equivalent alternatives $(i, k) \in N$, with respect to a given set of weighted criteria $M$, it holds for any $l \in N$, that $(i \succ l) \Leftrightarrow(k \succ l)$.

Condition 1 refers to any pair of equivalent alternatives, thus having the same amount of imprecision, and their preference situation with respect to a third alternative. In this way, if two alternatives are equivalent, then they should hold the same relation with any other alternative. Hence, we are basically looking at triplets of alternatives, examining the different situations that can emerge when making independent pairwise comparisons.

A natural situation to consider after the one depicted in Fig. 1, is one where two of the alternatives differ in their amount of imprecision, as shown in Fig. 2a. Say $k$ has greater imprecision than $i$ : in particular, $i$ and $k$ are partially equivalent. Then, if $k$ is preferred to a third alternative $l$, it should hold that $l$ cannot be preferred to $i$ due to the fact that $i$ has lower imprecision than $k$, and thus, that $i$ is either preferred or indifferent to $k$ under partial equivalence. The relative situation illustrating this second case is shown in Fig. 2b, taking into account that the third alternative can have any amount 


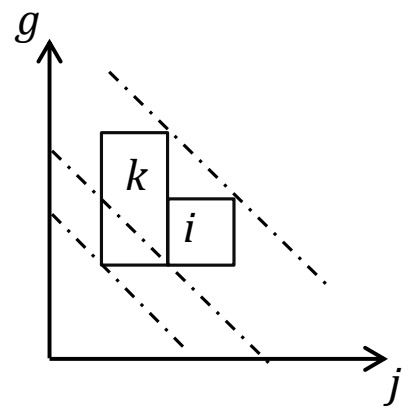

(a)

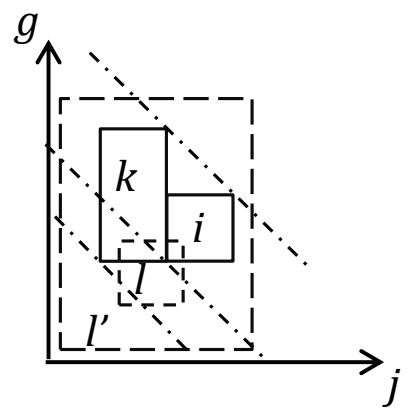

(b)

Figure 2: (a) Relative situation for alternative $i$ and a more imprecise one $k$, and (b) their preference situation regarding a third alternative $l$.

of imprecision (e.g., taking $l^{\prime}$, which is a very uncertain alternative, as in Example 1).

Condition 2. Given a pair of partially equivalent alternatives $(i, k) \in N$, with respect to a given set of weighted criteria $M$, it holds for any $l \in N$, that $(k \succ$ $l) \Rightarrow \neg(l \succ i)$.

Now, a stronger version of condition 2 would require that if a strict preference situation of $k$ over $l$ holds, then it should also hold that $i$ is strictly preferred to $l$.

Condition 3. Given a pair of partially equivalent alternatives $(i, k) \in N$, with respect to a given set of weighted criteria $M$, it holds for any $l \in N$, that $(k \succ$ $l) \Rightarrow(i \succ l)$.

Hence, under the (weak) Condition 2, if the alternative having greater imprecision is strictly preferred to a third alternative, then the alternative with less imprecision should at least not be strictly preferred by that third alternative. In this way, $i$ should be at least as preferred as $l$, where either $i \succ l$ or $i \sim l$. On the other hand, under the (stronger) version of Condition 3 , it should be true that $i \succ l$.

Therefore, Condition 2 refers to a concrete situation for any pair of partially equivalent alternatives $i, k \in N$ and its relation to a third alternative $l \in N$, which should satisfy a weak form of restricted semi-transitivity, such that $(i \succ k$ or $i \sim k)$ and $k \succ l$ imply that $\neg(l \succ i)$. In the same line, Condition 3 refers to a stronger requirement under the same restricted case of partial equivalence, where strict preference relations should satisfy a weak form of restricted transitivity, such that ( $i \succ k$ or $i \sim k$ ) and $k \succ l$ imply that $i \succ l$.

The three conditions for soundness can be used to evaluate pairwise preference estimation procedures on the different MCDM methods.

\section{Assessing the preference-consistency of imprecise MCDM}

Regarding the MCDM methods examined in Section 3 and how their pairwise comparison process develops, we can evaluate their performance on the basis of the soundness conditions presented in the previous Section 4.

\subsection{Distance-based performance}

Regarding distance-based methods, it can be shown that the TOPSIS method fulfills all three Conditions 1-3, whereas VIKOR does not necessarily fulfill Conditions 2-3.

For TOPSIS and VIKOR, it trivially follows that they fulfill Condition 1 , as for any pair of equivalent alternatives $i, k \in N, i \succ l$ holds for any $l \in N$ if, and only if, $k \succ l$ also holds, because the distance of $i, k$ to the positive (PIS) and negative (NIS) prototypes will obtain exactly the same values.

Regarding TOPSIS and Condition 2, it can be shown that it will always hold that if $i \succ k$ and $k \succ l$, then it cannot occur that $l \succ i$.

Proposition 1. The pairwise preference estimation procedure of the TOPSIS method (7) necessarily verifies Condition 2.

Proof. Under the relative situation of test 2, where $i \succ k$, there are two cases where it can hold both that $k \succ l$ and $l \succ i$. On the one hand, if $\mu_{l}^{U}>\mu_{k}^{U}$, then it has to be that $\mu_{l}^{L}<\mu_{k}^{L}$ for $k \succ l$ to be a possible outcome. Then, for $k \succ l$ to hold true, it has to hold that $R C_{k}>\frac{1}{2}$, and at the same time, for $l \succ i$ to hold true, it has to hold that $R C_{i}<\frac{1}{2}$, which cannot simultaneously hold, because by construction, $R C_{i}>R C_{k}$. On the other hand, if $\mu_{l}^{U}<\mu_{k}^{U}$, then it has to be that $\mu_{l}^{L}>\mu_{i}^{L}$ for $l \succ i$ to be a possible outcome. So, for $k \succ l$ to hold true, it has to hold that $R C_{k}>R C_{l}$, and at the same time, for $l \succ i$ to hold true, it has to hold that 
$R C_{l}<R C_{i}$, which cannot simultaneously hold because again, $R C_{i}>R C_{k}$.

Therefore, it directly follows from Proposition 1 that under the same relative situation of $i, k, l \in N$, if $i \succ$ $k$ and $k \succ l$, then it also holds that $i \succ l$, which corresponds with Condition 3.

In the case of the VIKOR method, it is enough to recall Example 1 as a counter-example for both Conditions 2 and 3. That is, being $i$ and $k$ partially equivalent, where $k \succ l$, it can hold that $l \succ i$.

In consequence, Conditions 1-3 illustrate different issues that deserve close attention, as they can be problematic for proper interval-valued multi-criteria preference modeling. In particular, it is observed that the specific pairwise preference outcome of the interval methods explored here depends entirely on the specific procedure that is used for ranking interval numbers (although the TOPSIS method overcomes this aspect by means of a uniquely valued distance measure for interval scores). In consequence, the analyst or decision maker has to determine how to obtain coherent results, by defining a different set of rules for comparing interval numbers with the VIKOR method.

\subsection{WOD performance}

Examining Conditions 1-3, it can be shown that the WOD method necessarily satisfies the first two (Condition 1 follows directly, while it has already been shown that the WOD method obtains a semitransitive order [7], thus verifying Condition 2), as well as Condition 3, irrespective of the particular selection of thresholds $\beta$ and $\gamma$.

Proposition 2. Given alternatives $i, k, l \in N$ and weights $w \in[0,1]^{m}$, such that $i$ and $k$ are partially equivalent, the pairwise preference estimation procedure of the WOD method (14)-(22) fulfills Condition 3.

Proof. Under the relative situation of test 3, $i$ and $k$ verify a partial overlapping situation, such that $i \succ k$ holds only if Eq. (15) is greater than $\beta$. Then two cases have to be considered: case 1 consists in $k$ and $l$ holding a partial overlapping situation, where it follows that $k \succ l$ and $i \succ l$ hold true, as the likelihood estimation for both is greater than the one of $i \succ k$, and hence greater than $\beta$. Case 2 consists in $k$ and $l$ holding a complete overlapping situation. In that case, if $i$ and $l$ are partially overlapping, then $i \succ l$ necessarily holds true following the same argument as before. But if $i$ and $l$ are completely overlapping, then we have two possible situations. One is that $Z_{k l}^{U} \neq \emptyset$, where $l$ has less imprecision, and the other is that $Z_{l k}^{U} \neq \emptyset$, where $l$ has the greatest imprecision. For the former, if $k \succ l$, then $R(k, l)>\gamma$ according to Eq. (22), such that $V\left(Z_{k l}^{U}\right)>$ $\gamma V\left(Z_{k l}^{L}\right)$. Due to the fact that $k$ and $i$ are partially equivalent under Eq. (2), then $V\left(Z_{i l}^{U}\right)=V\left(Z_{k l}^{U}\right)$ and $V\left(Z_{i l}^{L}\right)<V\left(Z_{k l}^{L}\right)$. Thus, the likelihood ratio for $i \succ l$ is higher than the one for $k \succ l$, as $R(i, l)>R(k, l)>\gamma$ and $k \succ l \Rightarrow i \succ l$. For the latter $\left(Z_{l k}^{U} \neq \emptyset\right)$, if $k \succ l$, then $R(l, k)<\gamma$, such that $V\left(Z_{l k}^{U}\right)<\gamma V\left(Z_{l k}^{L}\right)$. Then, given the partial equivalence between $k$ and $i$, it holds that $V\left(Z_{l i}^{U}\right)=V\left(Z_{l k}^{U}\right)$ and $V\left(Z_{l i}^{L}\right)>V\left(Z_{l k}^{L}\right)$. Thus, the likelihood ratio for $i \succ l$ is less than the one for $k \succ l$, as $R(l, i)<R(l, k)<\gamma$, and $k \succ l \Rightarrow i \succ l$.

Therefore, the WOD method verifies Conditions 13 , irrespective of the particular selection of free parameters $\beta$ and $\gamma$.

\section{Conclusion}

Three fundamental soundness conditions are suggested for imprecise multi-criteria decision modeling. Those conditions have been proven to be useful for examining the appropriateness of different models that are commonly used for MCDM with imprecise data. As a result, while Condition 1 is satisfied by all the different methods explored here, the other two conditions are not that easily verified.

Taking distance-based methods, the TOPSIS fulfills all the suggested tests, aided by the use of an explicit distance measure (chosen by the decision analyst) which simplifies the imprecise reasoning procedures by summarizing the available information for each alternative into precise, uniquely valued, multi-criteria scores (with the corresponding loss of information that such a simplification entails). On the other hand, although the VIKOR method allows a more detailed estimation of pairwise preferences, it fails to necessarily fulfill Condition 2, and it also fails when tested against Condition 3. Lastly, when testing the WOD method, it fulfills all tests.

In conclusion, the VIKOR method seems to present the most important anomaly, as it fails to verify Condition 2. For future research, it remains to examine the extent in which the anomaly for the VIKOR method may occur, e.g., by simulating random data and measuring the frequency of such anomalous outcomes. In this sense, it is also relevant to study applications of the different methods in real-world problems, evaluating the impact that the proposed conditions may have on decision-making. 
Besides, for future research, outranking procedures should also be tested, with respect the proposed conditions, or perhaps other conditions that can also be added to the soundness test here suggested. These conditions should also be extended for nonadditive multi-criteria methods, by means of general aggregation operators (as initially explored in [5]), also studying under a more general framework the conditions given for equivalence and partial equivalence.

\section{References}

[1] B. Ashtiani, F. Haghighirad, A. Makui, G. Montazer, Extension of fuzzy TOPSIS method based on interval-valued fuzzy sets, Applied Soft Computing 9 (2009) 457-461.

[2] D. Bouyssou, T. Marchant, M. Pirlot, A. Tsoukiàs, P. Vincke, Evaluation and Decision Models with Multiple Criteria, Springer, New York, 2006.

[3] C. Chen, Extensions of the TOPSIS for group decision-making under fuzzy environment, Fuzzy Sets and Systems 114 (2000) 1-9.

[4] T. Chen, C. Tsao, The interval-valued fuzzy TOPSIS method and experimental analysis, Fuzzy Sets and Systems 159 (2008) 1410 - 1428.

[5] C. Franco, J. Hougaard, K. Nielsen, Learning preferences and attitudes by multi-criteria overlap dominance and relevance functions, Applied Soft Computing 67 (2018) 641-651.

[6] P. Gupta, M. Mehlawat, N. Grover, Intuitionistic fuzzy multi-attribute group decision-making with an application to plant location selection based on a new extended VIKOR method, Information Sciences 370-371 (2016) 184-203.

[7] J. Hougaard, K. Nielsen, Weighted Overlap Dominance - a procedure for interactive selection on multidimensional interval data, Applied Mathematical Modelling 35 (2011) 3958-3969.

[8] G. Jahanshahloo, F. H. Lotfi, M. Izadikhah, An algorithmic method to extend TOPSIS for decision-making problems with interval data, Applied Mathematics and Computation 175 (2006) 1375-1384.

[9] P. Meyer, A. Olteanu, Handling imprecise and missing evaluations in multi-criteria majorityrule sorting, Computers and Operations Research 110 (2019) 135-147.
[10] S. Opricovic, G. Tzeng, Compromise solution by MCDM methods: A comparative analysis of VIKOR and TOPSIS, European Journal of Operational Research 156 (2004) 445-455.

[11] S. Opricovic, G. Tzeng, Extended VIKOR method in comparison with outranking methods, European Journal of Operational Research 178 (2007) 514-529.

[12] M. Sayadi, M. Heydari, K. Shahanaghi, Extension of VIKOR method for decision making problem with interval numbers, Applied Mathematical Modelling 33 (2009) 2257-2262.

[13] P. Yu, A class of solutions for group decision problems, Management Science 19 (1973) 936946.

[14] L. A. Zadeh, Fuzzy sets, Information and Control 8 (1965) 338-353.

[15] S. Çali, S. Balaman, A novel outranking based multi criteria group decision making methodology integrating ELECTRE and VIKOR under intuitionistic fuzzy environment, Expert Systems with Applications 119 (2019) 36-50. 